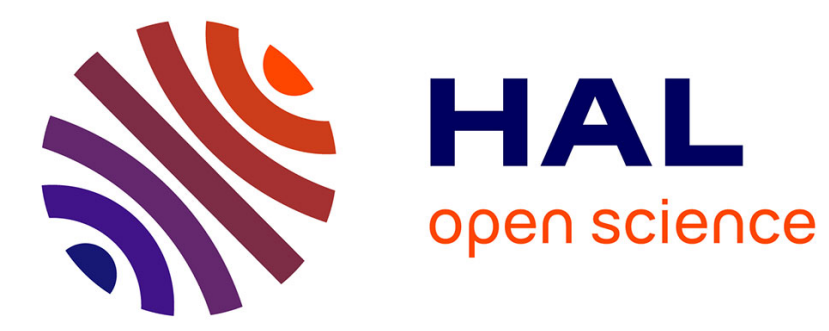

\title{
Psychanalyse et antipsychiatrie
}

\author{
Alain Vanier
}

\section{To cite this version:}

Alain Vanier. Psychanalyse et antipsychiatrie. Topique - Revue freudienne, 2004, Psychanalystes et psychiatres en France, 88, pp.79-85. 10.3917/top.088.0079 . hal-01524054

\section{HAL Id: hal-01524054 https://hal.science/hal-01524054}

Submitted on 2 Jun 2017

HAL is a multi-disciplinary open access archive for the deposit and dissemination of scientific research documents, whether they are published or not. The documents may come from teaching and research institutions in France or abroad, or from public or private research centers.
L'archive ouverte pluridisciplinaire HAL, est destinée au dépôt et à la diffusion de documents scientifiques de niveau recherche, publiés ou non, émanant des établissements d'enseignement et de recherche français ou étrangers, des laboratoires publics ou privés. 


\title{
PSYCHANALYSE ET ANTIPSYCHIATRIE
}

\author{
Alain Vanier \\ L'Esprit du temps | Topique
}

\begin{abstract}
2004/3 - no 88
pages 79 à 85
\end{abstract}

ISSN 0040-9375

Article disponible en ligne à l'adresse:

http://www.cairn.info/revue-topique-2004-3-page-79.htm

Pour citer cet article :

Vanier Alain , «Psychanalyse et antipsychiatrie »,

Topique, 2004/3 no 88, p. 79-85. DOI : 10.3917/top.088.0079

Distribution électronique Cairn.info pour L'Esprit du temps.

(c) L'Esprit du temps. Tous droits réservés pour tous pays.

La reproduction ou représentation de cet article, notamment par photocopie, n'est autorisée que dans les limites des conditions générales d'utilisation du site ou, le cas échéant, des conditions générales de la licence souscrite par votre établissement. Toute autre reproduction ou représentation, en tout ou partie, sous quelque forme et de quelque manière que ce soit, est interdite sauf accord préalable et écrit de l'éditeur, en dehors des cas prévus par la législation en vigueur en France. II est précisé que son stockage dans une base de données est également interdit. 


\title{
CA I R N
}

chercher : repérer : avancer

Cet article est disponible en ligne à l'adresse :

http://www.cairn.info/article.php?ID_REVUE=TOP\&ID_NUMPUBLIE=TOP 088\&ID_ARTICLE=TOP 0880079

\section{Psychanalyse et antipsychiatrie}

\author{
par Alain VANIER
}

\section{| L'Esprit du Temps | TOPIQUE}

\section{$2004 / 3-N^{\circ} 88$}

ISSN 0040-9375 | ISBN 2-8479-5040-0 | pages 79 à 85 conditions générales d'utilisation du site ou, le cas échéant, des conditions générales de la licence souscrite par votre établissement. Toute autre reproduction ou représentation, en tout ou partie, sous quelque forme et de quelque manière que ce soit, est interdite sauf accord préalable et écrit de l'éditeur, en dehors des cas prévus par la législation en vigueur en France. Il est précisé que son stockage dans une base de données est également interdit. 


\section{Psychanalyse et antipsychiatrie ${ }^{1}$}

Alain Vanier

Ce texte est avant tout le témoignage d'un trajet qui me conduisit, stagiaire en psychologie, puis vacataire, à participer, dans les années 70, au travail et à la vie de l'École expérimentale de Bonneuil-sur-Marne que fonda Maud Mannoni, celui d'une rencontre initiale avec la folie et ses institutions, qui me fit passer, paradoxalement, de l'antipsychiatrie à la psychiatrie, sans que, pour autant, le vif de cette première expérience ne cesse de marquer mon approche de ces questions. Revenir sur tout cela plus de trente ans après me donne l'occasion d'essayer de préciser comment ce moment des relations complexes entre psychanalyse et psychiatrie manifeste, avant tout, quelque chose de cette division qui nous traverse quand, psychanalystes, nous prenons part aux pratiques institutionnelles en psychiatrie. Après coup, ce peut être aussi la possibilité de comprendre ce qui fait que le surgeon français le plus connu de l'antipsychiatrie, l'expérience de Bonneuil, s'est produit dans une rencontre entre antipsychiatrie et psychanalyse, alors que dans d'autres pays, au contraire, c'est contre ou en dehors de la psychanalyse que s'est développée l'antipsychiatrie.

Dans les années de l'après-guerre, des pratiques nouvelles se proposant de remanier la psychiatrie vont voir le jour, de la psychothérapie institutionnelle à la politique de secteur, l'antipsychiatrie représentant la pointe extrême et radicale de ce mouvement. Cette contestation de l'institution asilaire trouve sa source aussi bien dans l'expérience des camps de la seconde guerre mondiale, les travaux contemporains comme ceux de Michel Foucault, que dans les possibilités qu'ouvre la découverte des premiers neuroleptiques. Nombre de ces

1. Texte repris d'une communication à la dixième rencontre de l'Association internationale d'Histoire de la Psychanalyse, Psychanalystes et psychiatres : une longue et complexe histoire, Centre Sèvres, Paris, 21-24 juillet 2004.

Topique, 2004, 88, 79-85. 
avancées s'appuieront sur la psychanalyse et sur l'œuvre de Lacan, ce qui est quelque peu paradoxal. On peut ici rappeler le passé de psychiatre de Lacan, repérable aussi bien dans l'exercice de la présentation de malades, dans l'affirmation réitérée de sa dette à l'égard de de Clérambault, ou dans les propos qu'il peut tenir, en 1967, aux internes en psychiatrie quand il leur reproche de se soucier trop de leur analyse et pas assez de l'approche clinique de leurs patients. Cet ancrage de Lacan dans la psychiatrie sera l'occasion, lors de la mise en place de la Section clinique en 1975, d'un conflit avec Maud Mannoni. Or, cette irruption de l'antipsychiatrie en France se produisit d'une façon originale à laquelle reste attaché avant tout son nom. Elle organisait en 1967 avec Ginette Raimbault un congrès intitulé Enfance aliénée ${ }^{2}$ qu'elle avait voulu placer sous l'égide de Lacan et de Winnicott. Mais celui-ci, pour des raisons diverses, dont, semble-t-il, la politique du mouvement analytique n'était pas absente, ne vint pas, mais adressa au congrès un texte et deux personnes, dont l'un était son élève, David Cooper et Ronald Laing. Auparavant, Winnicott, chez qui Mannoni était en contrôle, lui avait conseillé d'aller visiter Kingsley Hall. Selon elle, l'intervention de ces deux Anglais hors normes choqua le public Français, à l'exception de Lacan, qui le fit savoir dans la conclusion qu'il donna à ces journées. Parallèlement, dans une tonalité beaucoup plus politique que le mouvement anglo-saxon, Franco Basaglia développait en Italie une mise en question de la situation asilaire.

En 1969, Maud Mannoni crée, avec Robert Lefort, Rose-Marie et Yves Guérin, l'École expérimentale de Bonneuil-sur-Marne, «lieu-dit d'antipsychiatrie », pour accueillir des enfants autistes, psychotiques, débiles, arrachés à l'asile ou simplement exclus du système scolaire. Le lien immédiat avec la psychanalyse dans l'expérience française fut aussi ce qui la tempéra, lui donna son originalité et son ampleur, et lui permit aussi d'être moins sensible aux mouvements de la mode qui firent basculer quelqu'un comme Ronald Laing vers ce qu'il appellera full psychiatry, un certain retour à une conception psychiatrique somme toute assez traditionnelle, malgré les diverses modifications qu'il entendait y introduire.

En effet, pour Maud Mannoni, la psychose de l'enfant ne pouvait être réduite à un strict produit des agencements familiaux ou du discours social. Mais ceuxci avaient comme effet de figer ce qu'il y a de dynamique dans la psychose, conformément aux propositions freudiennes sur le délire. Pour elle, il s'agissait de laisser se remettre en mouvement ce que la psychiatrisation figeait. On peut évoquer ici Foucault opposant la démarche objective des sciences humaines à la psychanalyse où «ce n'est pas seulement la connaissance qu'on a de l'homme qui est engagée, mais l'homme lui-même ${ }^{3} »$.

2. Enfance aliénée (1967), Paris, Denoël, 1984.

3. M. Foucault, Les mots et les choses, Paris, Gallimard, 1966, p. 387. 
Quant à l'effet du discours familial, elle nuancera, et de plus en plus au fur et à mesure de l'avancée de l'expérience, ce que certains théoriciens anglais ou américains ont pu concevoir comme mécanisme de production d'une psychose. En effet, son premier ouvrage, L'enfant arriéré et sa mère ${ }^{4}$, développe la question de la prise de l'enfant dans le fantasme maternel et ses conséquences sur la structure du sujet. Elle précisera ultérieurement qu'il ne faut pas sousestimer non plus la façon dont le trouble de l'enfant façonne une «mère de psychotique » selon le mot malheureux en circulation à cette époque.

L'interposition ou la mise en place du fantasme comme organisateur de la structure subjective, change d'une certaine manière la donne d'une conception par trop sociale de la folie qui peut conduire à une dérive paranoïaque du discours antipsychiatrique. En effet, le risque n'est pas nul de constituer à côté d'un dedans idéalisé de l'institution un dehors social ou parental, mauvais, persécutant, devant être rejeté à l'extérieur.

Certes, l'indistinction entre psychologie collective et psychologie individuelle, affirmée par Freud, peut fonder l'expérience antipsychiatrique, c'est-à-dire comme expérience devant subvertir toute adhésion radicale du sujet à une identité sociale. Ce que Mannoni conteste est l'effet du diagnostic et la façon dont cet épinglage est à sa manière une tentative d'inscrire le hors lien social de la psychose tout en fondant son exclusion. Pour elle, cette question est essentielle, ce qui ne veut pas dire décisive, car il y a une structure de la psychose qui ne réduit pas la folie à sa simple désignation.

Le mouvement antipsychiatrique permettra non seulement une mise en question des structures psychiatriques traditionnelles, mais donnera aussi un retentissement aux travaux mal connus du monde anglo-saxon (Palo Alto, Szasz, etc.). Son style particulier en France n'est pas sans rapport avec la place de la psychanalyse dans la vague des événements de l'époque. Pour Basaglia, par exemple, la psychanalyse était l'activité de quelques praticiens de ville, destinée à traiter les vapeurs bourgeoises mais ne s'occupant pas de la psychose du fait de la désocialisation qui l'accompagne. L'antipsychiatrie trouvait donc une autre source en Italie ${ }^{5}$. Ici, la mise en question de l'institution psychiatrique se fait dans une perspective marxiste. Il s'agit de montrer que «ce qu'il y a de fondamentalement erroné dans notre image de la folie est d'abord que c'est une image sociale, que l'asile n'est rien qu'une forme de la pression de la société sur ceux (toujours les pauvres au demeurant) qui y sont enfermés ». Il est certain que la folie et l'asilisation s'accompagnent toujours d'une désocialisation qui paupérise ceux qui sont enfermés. Mais cette désocialisation propre à la psychose, qui conduit le plus souvent le fou, quelle que soit son origine sociale, à rejoindre le sous-prolétariat, ne risque-t-elle pas d'annuler, de méconnaître la folie pour se contenter de l'inscrire dans la lutte des classes ? N'y a-t-il pas

4. M. Mannoni, L'enfant arriéré et sa mère, Paris, Seuil, 1964.

5. F. Basaglia (dir.) (1968), L'institution en négation, Paris, Seuil, 1970. 
là au fond une subtile stratégie qui permettrait de refouler, de nier ce qu'il y a de spécifique dans la psychose, et dans la manière dont elle interroge la «normalité »? S'agirait-il de faire taire ce qu'il y a d'insupportable, d'inassimilable à une conception marxiste de l'histoire, et qui tient à la folie ? En France, du fait de l'histoire de la psychiatrie et de l'importance du mouvement analytique, cette critique des institutions allait prendre une autre allure.

L'autre aspect qui singularise le mouvement antipsychiatrique français par rapport à ceux qui se sont développés en Angleterre et en Italie, est qu'il concerne les enfants. Or, la psychiatrie de l'enfant a longtemps été plus marquée par la psychanalyse que celle de l'adulte, et la psychanalyse de l'enfant s'est d'emblée inscrite dans un projet que l'on peut dire social, puisque comprenant une ambition prophylactique et une incidence éducative. Mais, dans cette perspective, pour Maud Mannoni, la ségrégation était redoublée : elle concernait la folie bien sûr, mais aussi l'enfance. Pour elle, ce monde de l'enfance de plus en plus coupé du monde de l'adulte, cette culture moderne de l'enfance Philippe Ariès avait montré que l'enfant dans des époques plus anciennes appartenait très tôt au monde adulte ${ }^{6}$ - était interprété comme une autre ségrégation.

Ainsi, «l'«antipsychiatrie»s'inscrit dans un projet plus polémique que politique», écrivait Pierre Fédida qui participa aux débuts de Bonneuil, «il s'agit moins de prendre le contre-pied de la psychiatrie traditionnelle que de dénoncer le jeu subtil des compromissions idéologiques et pseudo scientifiques qui entre dans le projet de la psychiatrie quel qu'elle soit ». En effet, ce que fait apparaître l'antipsychiatrie, c'est que du lien privilégié que la psychiatrie a noué avec la médecine, «il en est résulté une éthique de la norme qui valait aussi bien pour la santé que pour la morale et pour l'ordre social et politique ${ }^{7} \gg$. Si la psychanalyse pouvait soutenir ce questionnement, alors elle ne pouvait se suffire de l'introduction de quelques psychanalystes dans les institutions pour régler la question. Ce fut d'ailleurs l'expérience de Maud Mannoni avant la création de l'école de Bonneuil. Elle pratiqua dans divers lieux cliniques et constata les difficultés, voire, selon elle, l'impossibilité d'une pratique de la psychanalyse en institution ${ }^{8}$. Il s'agit d'en tirer les conséquences politiques, c'est-à-dire de prendre au sérieux la question qu'ouvre l'introduction de la psychanalyse dans une institution et les effets sur la structure institutionnelle, car la découverte freudienne subvertit les frontières couramment admises du normal et du pathologique.

C'est pourquoi, sans doute, en créant l'école expérimentale de Bonneuil en 1969, Maud Mannoni se réclamait du mouvement antipsychiatrique, mais en même temps s'en séparait tout comme elle prenait ses distances, en reconnaissant sa dette, à l'égard de la psychothérapie institutionnelle. Partant des

6. P. Ariès (1960), L'enfant et la vie familiale sous l'Ancien Régime, Paris, Seuil, 1973.

7. P. Fédida (1968), «Psychose et parenté », in Le concept et la violence, Paris, UGE, 1977.

8. M. Mannoni, Le psychiatre, son «fou» et la psychanalyse, Paris, Seuil, 1970. 
avancées des antipsychiatres, elle retenait la fonction de la ségrégation, de la folie comme statut, mais s'en écartait en ne soutenant pas qu'on peut résumer la folie à un simple effet social. À partir de la confrontation ou des tentatives d'articulations entre psychanalyse et antipsychiatrie, elle concevait le projet original qu'elle allait mettre en place. De son expérience de praticienne en institution, elle tirait la conclusion qu'il n'est pas possible d'introduire la psychanalyse comme spécialité complémentaire dans le champ de la psychiatrie, car, d'une part, la parole libérée par la cure n'est pas supportée par le lieu institutionnel, et d'autre part l'analyste pour le patient est suspect d'une complicité avec les forces répressives qui l'ont interné. Il convient alors de repenser l'espace institutionnel et son articulation à la psychanalyse.

L'autre question qui demeure ouverte à notre époque, touche à la question du statut de la folie en tant que telle. Existe-t-il des structures anhistoriques qui traverseraient les temps de façon immuable, mais qui trouveraient à s'exprimer dans les champs culturels du moment, ou bien une notion comme celle de folie n'a-t-elle de sens que rapportée à une norme, ce que Foucault a cherché à montrer avec la notion de perversion ou avec celle d'homosexualité, n'est-elle que le produit d'un dispositif discursif propre à une époque historique donnée? Plus récemment, Édouard Zarifian peut écrire : «C'est la psychiatrie qui définit la folie. Ne pas rencontrer la psychiatrie, c'est éviter l'étiquette indécollable de «fou ». Le rôle de la psychiatrie, c'est d'officialiser ce statut ${ }^{9} »$. Il faut remarquer que la clinique sur ce plan n'est pas sans nous laisser dans une certaine perplexité ${ }^{10}$.

Mais le «problème de la ségrégation n'est pas un problème purement politique ; au cœur de chacun de nous, il y a place pour le rejet de la folie, c'està-dire pour le rejet de notre propre refoulé» ${ }^{11}$. Ainsi, à Bonneuil, la psychanalyse se trouve convoquée, dans sa dimension de subversion d'un savoir, pour soutenir cette interrogation et ses effets dans la pratique. Au niveau du principe de son fonctionnement, il ne se pratique pas de cure psychanalytique dans l'école de Bonneuil, ni d'analyse institutionnelle, c'est littéralement « un lieu pour vivre». Les enfants poursuivent leurs analyses à l'extérieur de l'institution, et le malaise des soignants est renvoyé à l'éventuelle interrogation psychanalytique que la rencontre avec ces enfants en difficulté peut ouvrir chez eux. Le lieu d'une analyse possible à l'extérieur d'une institution, dont l'analyste n'ait pas à rendre compte à l'intérieur de celle-ci, maintient un espace «permettant l'expression du négatif envers l'institution, authentifiant ainsi ce qu'il y a de répétitif et de transférentiel ${ }^{12} »$. Et Robert Lefort ajoute : «C'est une dimension qui est

9. E. Zarifian, Les jardiniers de la folie, Paris, Odile Jacob, 1988.

10. Voir A. Vanier, «À propos d'un cas de «guérison» paradoxale par la psychanalyse», L'Évolution psychiatrique, vol. 66, n¹, Paris, Elsevier, Janvier-Mars 2001.

11. M. Mannoni, Le psychiatre, son «fou» et la psychanalyse, op. cit., p. 243.

12. R. Lefort, «Discours de l'institution et sujet du discours », in Maud Mannoni, Éducation impossible, op. cit. 
évidemment très atténuée, voire absente, lorsque l'analyste est à l'intérieur de l'institution et est identifié à elle par le sujet parlant : ce qui le fait alors s'adresser à une réalité le poussant sur le versant paranoïaque du discours. »

Bonneuil, au départ, s'est donc voulu lieu de vie et non institution soignante. Pourtant, la pratique quotidienne à Bonneuil se soutient d'une approche psychanalytique : la psychanalyse est convoquée comme outil de lecture de la vie de l'institution. Cette lecture intervient dans l'après-coup et permet de questionner les trajets individuels et les avatars institutionnels. C'est ce mouvement de vaet-vient permanent entre pratique et théorie - en particulier dans les groupes de travail - qui caractérise la place de la psychanalyse à Bonneuil. La place des psychanalystes y est donc paradoxale : ce n'est pas la pratique qui les institue comme tels qu'ils viennent y poursuivre, mais c'est d'elle que se spécifie leur place en ce lieu et le trajet qu'ils viennent faire avec les enfants en difficulté.

Il faut regarder Bonneuil comme l'essai d'un mode conséquent d'articulation de la psychanalyse avec la psychiatrie, et peut-être ne pas en oublier la leçon, à savoir que la psychanalyse ne peut pas entrer dans le champ psychiatrique comme une technique parmi d'autres, une parmi les psychothérapies. Que la référence ou l'idée psychanalytique dans une institution psychiatrique ne puisse être sans conséquences sur l'organisation institutionnelle elle-même, à cause précisément de la théorie de l'institution, de la théorie du groupe que suppose la psychanalyse. Elle suppose le maintien d'une tension à faire travailler entre deux pôles « antipathiques».

Enfin, sans doute convient-il de souligner que c'est une femme, une Étrangère au carrefour de plusieurs cultures, ni psychologue, ni psychiatre, une laïque donc, qui introduisit l'antipsychiatrie en France et lui donna le style particulier qu'elle eut ici.

Alain VANIER

47 avenue Paul Doumer

75116 Paris 


\section{BIBLIOGRAPHIE}

ARIÈS P. (1960), L'enfant et la vie familiale sous l'Ancien Régime, Paris, Seuil, 1973. BASAGLIA F. (dir.) (1968), L'institution en négation, Paris, Seuil, 1970.

COOPER D. (1970), Psychiatrie et anti-psychiatrie, Paris, Seuil, 1970.

COOPER D. Enfance aliénée (1967), Paris, Denoël, 1984.

FEDIDA P., Le concept et la violence, Paris, UGE, 1977.

FOUCAULT M., Folie et déraison. Histoire de la folie à l'âge classique, Paris, Gallimard, $1961,1972$.

FOUCAULT M., Les mots et les choses, Paris, Gallimard, 1966.

LAING R.D. (1961-1969), Soi et les autres, Paris, Gallimard, 1971.

LAING R.D. et ESTERSON A. (1964), L'Équilibre mental, la folie et la famille, Paris, Maspéro, 1970.

LEFORT R., «Discours de l'institution et sujet du discours », in Maud Mannoni, Éducation impossible, Paris, Seuil, 1973.

MANNONI M., L'enfant arriéré et sa mère, Paris, Seuil, 1964.

MANNONI M., Le psychiatre, son «fou » et la psychanalyse, Paris, Seuil, 1970.

MANNONI M., Éducation impossible, Paris, Seuil, 1973.

MANNONI M., Un lieu pour vivre, Paris, Seuil, 1976.

VANIER A., «Trajet de Maud Mannoni », in Journal français de Psychiatrie, ${ }^{\circ} 10$, Paris, Érès, $2^{\mathrm{e}}$ trimestre 2000.

VANIER A., «À propos d'un cas de "guérison" paradoxale par la psychanalyse », in L'Évolution psychiatrique, vol. 66, $\mathrm{n}^{\circ}$ 1, Paris, Elsevier, janvier-mars 2001.

ZARIFIAN E., Les jardiniers de la folie, Paris, Odile Jacob, 1988.

\section{Alain Vanier - Psychanalyse et antipsychiatrie}

Résumé : Maud Mannoni introduisit l'antipsychiatrie en France, et lui donna un style particulier en l'articulant à la psychanalyse, dont témoigne l'expérience de l'École expérimentale de Bonneuil, qu'elle fonda en 1969.

Mots-clés : Antipsychiatrie - Psychanalyse et institution - Psychanalyse avec les enfants.

Alain Vanier - Psychoanalysis and Antipsychiatry

Summary : Maud Mannoni introduced antipsychiatry in France, and gave it a particular style ; the Experimental School of Bonneuil, she founded in 1969, is an evidence of this specificity.

Key-words : Antipsychiatry - Psychoanalysis and institution - Child psychoanalysis. 